Boletín de la Sociedad Geológica Mexicana

VOLUMEN 62, NÚM. 1, 2010, P. 187-198

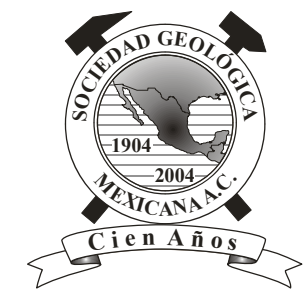

\title{
La pequeña minería del oro en Cuba: historia, metalogenia y perspectiva
}

\author{
Roberto Díaz Martínez ${ }^{1}$ \\ ${ }^{1}$ Instituto Superior Minero Metalúrgico de Moa, Las Coloradas, s/n, Moa, Holguín, C.P. 83320, Cuba. \\ *rdmart@ismm.edu.cu
}

\begin{abstract}
Resumen
La historia de la minería del oro en Cuba ha sido descrita en numerosos trabajos publicados, sin embargo no ha sido sistematizada acorde a las diferentes etapas del desarrollo socio-económico de la isla. Asimismo, se han elaborado clasificaciones y modelos conceptuales en el campo de la metalogenia del oro en Cuba, muchas de las cuales son profusas y no reflejan claramente los atributos esenciales que caracterizan las mineralizaciones de oro en la isla. Lo antes expuesto permitió desarrollar una investigación de carácter histórico-descriptivo sobre la base de una exhaustiva revisión, análisis e interpretación de la información disponible. La minería de oro en la isla se puede enmarcar en tres periodos históricos: colonial, neocolonial y revolucionario.

En la evolución geológica de Cuba se reconocen 4 episodios metalogenéticos principales de oro: (i) Aptiense-Campaniense, con la formación de mineralizaciones porfídicas de $\mathrm{Au}-\mathrm{Cu}$, de skarn de $\mathrm{Au}$ y epitermales de $\mathrm{Au}$ y Ag de baja y alta sulfuración; (ii) Campaniense tardío-Daniense temprano, donde se formaron mineralizaciones de oro de tipo orogénico y en listvenitas; (iii) Daniense-Eoceno medio, muy prolífero en mineralizaciones de oro en listvenitas (Cuba central) y asociadas al arco de islas volcánicas del Paleógeno (sur de Cuba oriental); y (iv) Oligoceno-reciente, donde se han originado las mineralizaciones en sobreros de hierro a partir de la oxidación de yacimientos de menas sulfurosos y placeres. La futura minería del oro en Cuba es muy atractiva, pero a pequeña y mediana escala.
\end{abstract}

Palabras clave: Pequeña minería, oro, historia, metalogenia, Cuba.

\begin{abstract}
The history of gold mining in Cuba has been described in numerous published works; however, it has not been systematized in accordance with the socio-economic developments of the island. Several classifications and conceptual models have been presented on the gold metallogeny in Cuba, but they are not clear and do not plainly reflect the essential attributes that characterize gold mineralization on the island. The aforementioned circumstances justified a historical-descriptive research project based on an exhaustive literature review, and the analysis and interpretation of the available information. Gold mining on the island extends over three historical stages: colonial, neocolonial and revolutionary.

Four main metallogenetic episodes for gold formation can be distinguished on the island of Cuba: (i) an Aptian-Campanian stage of mineralization with low- and high-sulfidation Au-Cu porphyries, Au skarns and Au-Ag epithermal deposits; (ii) a late Campanianearly Danian episode that hosts orogenic-type gold mineralization and listvenites; (iii) very prolific Danian-middle Eocene gold mineralization in listvenites in Central Cuba and in Palaeogene volcanic island arc rocks in the southern part of eastern Cuba; and lastly, (iv) an Oligocene-Recent mineralization stage in which surface gossans are produced by the oxidation of sulfide ores and placers. The future of gold mining in Cuba is very attractive, but at a small to medium scale.
\end{abstract}

Keywords: Small-scale mining, gold, history, metallogeny, Cuba. 


\section{Introducción}

La minería del oro en Cuba, a pesar de su limitado impacto socioeconómico nacional, posee una rica tradición popular legada por su propia historia, que va desde los tiempos de la conquista hasta la actualidad. Numerosos trabajos reseñan los datos históricos de esta actividad en la isla, resaltando los periodos colonial y neocolonial (Calvache-Dorado, 1944; Soto-González, 1981; Font-Cruz, 1985). Sin embargo la etapa revolucionaria, muy rica en investigaciones científicas, al menos en su último periodo, aún no se ha escrito.

Las mineralizaciones de oro en Cuba se localizan principalmente en una faja metalogenética de aproximadamente $400 \mathrm{~km}$, que se extiende desde la porción sur de Santa Clara hasta el norte de Holguín (Figura 1).

En correspondencia con la modelación geológica de yacimientos minerales (Ariosa-Iznaga y Díaz-Martínez, 2001; Ariosa-Iznaga, 2002; Atkinson, 2003) y sobre la base del incremento del conocimiento geológico de la isla se han elaborado diferentes clasificaciones (López-Kramer et al., 1998; Proenza y Melgarejo, 1998; Rodríguez-Vega y Díaz-Martínez, 2001; Rodríguez-Romero, 2001) y modelaciones (Lavandero et al., 2001; Capote-Marrero et $a l ., 2009)$ relacionadas con la metalogenia del oro en Cuba.
Sin embargo, muchas de estas clasificaciones son profusas y en ocasiones se limitan solamente a describir tipos genéticos de depósitos, sin una contextualización clara sobre la base de la edad y ambiente geológico donde se encuentran los yacimientos.

Los aspectos antes mencionados fundamentan la necesidad de realizar la presente revisión, cuyo objetivo principal es proponer los principales estadios metalogenético del oro en Cuba como herramienta para su exploración geológica.

\section{Métodos y materiales}

En la investigación que se presenta se aplicó el método descriptivo de la investigación científica, basándose en la revisión, análisis y generalización de la información geológica disponible sobre las mineralizaciones de oro en Cuba. La misma contempló las fases o etapas heurística y hermenéutica del procesamiento de información.

La fase heurística consistió en la búsqueda y recopilación de las fuentes de información sobre la minería, los rasgos geológicos, mineralógicos y geoquímicos de los diferentes tipos de depósitos, manifestaciones e indicios de oro en Cuba.

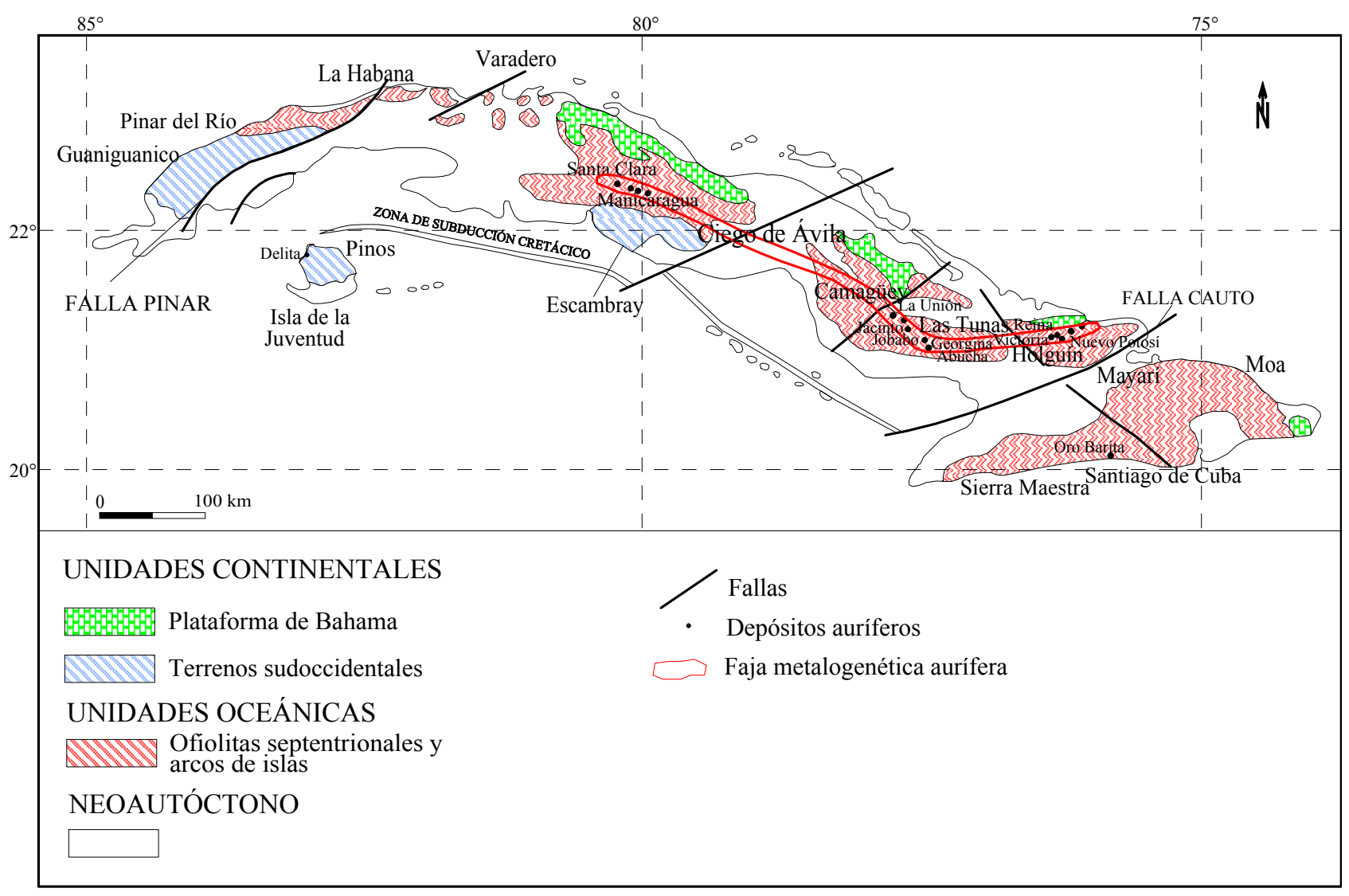

Figura 1. Principales depósitos de oro de Cuba. 
La fase hermenéutica consistió en clasificar, analizar e interpretar las diferentes fuentes bibliográficas consultadas, permitiendo sistematizar la información disponible sobre la base de la evolución histórica de la minería del oro, los principales rasgos metalogenéticos del oro (edad del evento de la mineralización, unidades geotectónicas y regiones del país donde se revelan, tipología de depósito, principales rasgos composicionales) y la perspectiva de la minería del oro en Cuba.

\section{Resultados}

\subsection{Breve historia de la minería del oro en Cuba}

La minería del oro en Cuba estuvo estrechamente ligada a la conquista de España y fue practicada en sus inicios por los aborígenes de la isla. La misma se caracterizó por ser recolectora, razón por la cual Calvache-Dorado (1944) la denominó "Minería Taína"; posteriormente, debido a los descubrimientos arqueológicos, se le denominó "Minería Indígena" (Soto-González, 1981).

La isla de Cuba, a pesar de ser un país relativamente pequeño, tiene una larga trayectoria minera dado sus recursos metalogenéticos (Proenza y Melgarejo, 1998). Sin embargo, la extracción de oro, desde sus inicios, nunca ha tenido un impacto económico digno de considerar (Rodríguez-Vega y Díaz-Martínez, 2001). A la llegada de los conquistadores españoles, la isla de Cuba contaba con una población de 112,000 habitantes (Torres-Cuevas y Loyola-Vega, 2001), los cuales practicaban la llamada minería recolectora ("Minería Indígena"). En esta etapa las pepitas de oro se obtenían directamente de los aluviones de los ríos (ej. Gibara, Jobabo, Guáimaro, Agabama, Arimao y Guaracabuya), con el objetivo de elaborar las guayzas que mostraban durante sus rituales.

En el diario de Colón aparecen 65 páginas dedicadas al oro, entre el 12 de octubre de 1492, en que desembarcó en la primera isla y el 17 de enero de 1493, en que regresó definitivamente a la península. De ahí la interrogante formulada por Pierre Villar: ¿Colón buscaba oro? (SánchezRodríguez, 2001). Aunque los aborígenes cubanos reconocían los ríos como los sitios idóneos para su recolección, no es hasta la llegada de los conquistadores que en Cuba existió una verdadera actividad minera; por tanto, actualmente se conocen tres periodos importantes en el desarrollo de la minería del oro en la isla: colonial, neocolonial y revolucionario.

\subsubsection{Periodo colonial (1511-1898)}

La conquista de los españoles trajo consigo la afanosa búsqueda del oro en Cuba, actividad esta que se caracterizó por una ambición desenfrenada, utilizando mano de obra indígena, lo que provocó la eliminación de la escasa población indígena existente, que nada recibía a cambio de su trabajo (Hamilton, 1998).
La etapa inicial de la colonización se caracterizó por la extracción de oro aluvionar, aunque se cree que en la región central de Las Villas, llamada en lengua Taína Cubanacán, los indios lo extraían de las rocas (Brauns, 1860).

Diego Colón, virrey de La Española, indagó acerca del potencial aurífero de la isla y envió a Diego Velázquez de Coellar en una expedición que desembarcó en 1510 en el extremo oriental de Cuba. Estando en Cuba ordenó a Pánfilo de Narváez realizar una exploración por toda la isla, encontrando oro en Bayamo, Jobabo, Guáimaro, Arimao, Agabama y Jaruco.

El inicio de la metalurgia del oro se remonta a finales de 1512 en Bayamo (en la actual provincia Granma) con la construcción de la primera fundición de oro en Cuba, la cual fue trasladada en abril de 1515 a la ciudad de Santiago de Cuba.

En los cuatro años siguientes a la conquista (1512-1515) se remitieron a la corona española remesas valoradas en 12,437 pesos en pago al impuesto denominado el quinto de la corona. Entre 1515 y 1538 estas remesas representaron una producción equivalente a 2,000,000 de pesos, aunque estimaciones de la época indican un valor de casi 3,000,000 de pesos (Soto-González, 1981).

En 1542 culminó la explotación del oro en Cuba e inició la minería del cobre, que tuvo por sede a Santiago del Prado, actual localidad de El Cobre (Torres-Cuevas y Loyola-Vega, 2001).

\subsubsection{Periodo neocolonial (1902-1958)}

En este periodo la pequeña minería del oro en Cuba se centró fundamentalmente en la región de Villa Clara y en Holguín, siendo esta última la de mayor importancia para el país. En la descripción geológica sobre las minas de oro en Cuba se incluyen las minas San Juan Bautista, Guajabales, La Caridad en Aguas Claras, entre otras.

En el periodo 1914-1919 se realizaron varios trabajos de exploración geológica en la región de Holguín con resultados positivos, lo que posibilitó que en la segunda mitad de la década del 30 del siglo XX se construyera una planta de beneficio, dirigida por el español Manuel Zamora y con la ayuda de su amigo Juan Bretones, quien se desempeñó como químico y geólogo (Font-Cruz, 1985).

Esta etapa se consideró la de mayor bonanza para las pequeñas empresas mineras con producciones ascendentes al medio millón de gramos de concentrados de oro, destinadas al mercado estadounidense de la época. La principal producción de oro provenía principalmente del yacimiento Nuevo Potosí, que cierra sus operaciones mineras a inicios de la segunda guerra mundial.

Durante el gobierno de Gerardo Machado y Morales (1925-1933), los vecinos del poblado de Aguas Claras en Holguín, conocedores del potencial aurífero de esta zona, se dedicaron a trabajar en los lavaderos de oro que ya se conocían con anterioridad. La búsqueda y extracción de pepitas de oro nativo se realizaba mediante el lavado de las arenas de los arroyos, cañadas y hasta del polvo de los 
caminos. Una vez obtenido el concentrado de oro rico en magnetita se amalgamaba añadiendo mercurio y tras su destilación quedaba listo para ser vendido a los árabes, quienes compraban todo el metal precioso que se obtenía de forma artesanal.

\subsubsection{Periodo revolucionario (1959-2009)}

La etapa inicial de este periodo centra su atención en la profundización del conocimiento geológico del país, la reevaluación de su potencial aurífero y la continuidad de la explotación y concentración del oro en la región de Holguín.

En el año 1962 se crea el Instituto Cubano de Recursos Minerales (ICRM) y reinician los trabajos de levantamientos geológicos a diferentes escalas, trabajos topográficos, geoquímicos y geofísicos, de perforación y laboreos mineros. Al frente de los mismos se encontraba el italiano Sergio Biscucia, que ya contaba con gran experiencia por sus trabajos anteriores en los depósitos de Reina Victoria, Guajabales, Nuevo Potosí y Agrupada en Aguas Claras.

En los primeros años de la revolución, los vecinos del poblado de Aguas Claras, decididos a reiniciar las operaciones mineras y reabrir la planta de beneficio de oro, crean una pequeña cooperativa, que solamente contaba con un camión de obras públicas. Un grupo de obreros se dedicó a repasar antiguas colas por mesas de concentración y a obtener oro por amalgamación. Estos obreros obtuvieron modestos resultados que ayudaron a aliviar la difícil situación económica de aquellos primeros años de revolución.

En la etapa comprendida entre 1962 y 1990 fueron estudiados la mayoría de los placeres de oro del distrito Holguín, resultando el pequeño placer Arroyo de Oro el más prometedor con recursos hipotéticos de $102 \mathrm{~kg}$ de Au nativo.

$\mathrm{Al}$ inicio de la década de los 90 se produce la apertura económica a la inversión extranjera en el sector de la minería en Cuba. Se crean las sociedades económicas mixtas Holmer Gold Mine S.A., Siboney S.A., Republic, Miramar, entre otras, desarrollando trabajos de exploración en áreas potencialmente perspectivas en todo el país. En el año 1996 la asociación económica Minería Siboney-Geominera S.A. descubre la manifestación de Au-Cu La Unión a 16 km al SSW de Sibanicú en la provincia de Camagüey. Los contenidos de oro oscilan entre 3.53 y $1.18 \mathrm{~g} / \mathrm{t}$.

En el año 1999 Spiteri Geological and Mining Consultants Inc. realiza estudios conceptuales en las vetas Beatriz, El Limón y Elena Sur en la región de Camagüey y en el año 2000 Carib Gold-Geominera S.A. llevó a cabo estudios económicos con vista a la puesta en marcha de un proyecto de explotación minera.

A partir del año 1995 la compañía australiana Rhodes Victoria S.A. asumió los estudios de exploración y evaluación de los yacimientos de oro del distrito Holguín, retirándose años más tarde por razones económicas.

La pequeña minería del oro en la región de Holguín ha sido practicada desde antaño por sus pobladores como alternativa para minimizar los efectos económicos de las diferentes crisis económicas por las que ha atravesado el país. Es así como en los últimos diez años se hace notoria la presencia de lavadores de oro en diferentes localidades del norte de Holguín. Los laboreos mineros ejecutados en el prospecto Reina Victoria durante la exploración geológica produjeron enormes pilas de material que actualmente son aprovechados por "jagueros", nombre asignado en Cuba a los bateadores de oro (Figura 2).

Esta actividad descontrolada y fuera de los marcos jurídicos de la isla ha provocado considerables daños al medio ambiente y un enriquecimiento ilegal de los pobladores de todas las localidades aledañas a los placeres (Figura 3).

En la localidad de Cuatro Palmas, a $4 \mathrm{~km}$ al sur del balneario turístico Guardalavaca, existe una fuerte tradición de la minería artesanal del oro; sus pobladores obtienen cantidades considerables de oro provenientes de los aluviones, suelos y zonas de alteración metasomática (Figura 4). En ocasiones se han encontrado pepitas, luego de las crecidas de los arroyos, de hasta 111 gramos.

Una última etapa se relaciona con las posibilidades reales de extracción de oro en áreas evaluadas, para lo cual el Grupo Geominsal perteneciente al Ministerio de la Industria Básica ha asumido la responsabilidad de dirigir el programa de oro en el primer decenio del siglo XXI en el país. Se tiene previsto la explotación a cielo abierto del yacimiento Golden Hill en Camagüey y Oro Barita en Santiago de Cuba y por vía subterránea del yacimiento Falcón en Placetas, provincia de Villa Clara.

\subsection{Metalogenia del oro en Cuba}

En Cuba se reconocen cuatro estadios evolutivos principales involucrados en el origen y distribución espaciotemporal de las mineralizaciones de oro, las cuales son descritas a continuación.

\subsubsection{Estadio Aptiense-Campaniense}

Durante el Aptiense-Campaniense la litosfera oceánica generada durante los procesos de génesis del rift continental y estructuración del "Protocaribe", en el Jurásico-Cretácico Inferior, fue subducida. En estas nuevas condiciones geodinámicas surge un complejo vulcano-plutónico asociado con el denominado arco de islas volcánicas del Caribe (Donnelly et al., 1990; Lebron y Perfit, 1993; Iturralde-Vinent, 1994).

Los materiales del arco Cretácico se distribuyen a lo largo de toda la isla (Díaz de Villalvilla y Dilla, 1985) siendo más frecuentes en la región Ciego de Ávila-CamagüeyLas Tunas. La manifiesta zonalidad de norte a sur de este arco permite diferenciar las regiones retro, axial y frontal del arco (Iturralde-Vinent, 1998), con predominio de mineralizaciones de oro en la zona axial.

La presencia de mineralizaciones de $\mathrm{Au}-\mathrm{Cu}$ en pórfidos y de oro en skarn y epitermal en la región Ciego de ÁvilaCamagüey-Las Tunas constituye un sólido argumento 


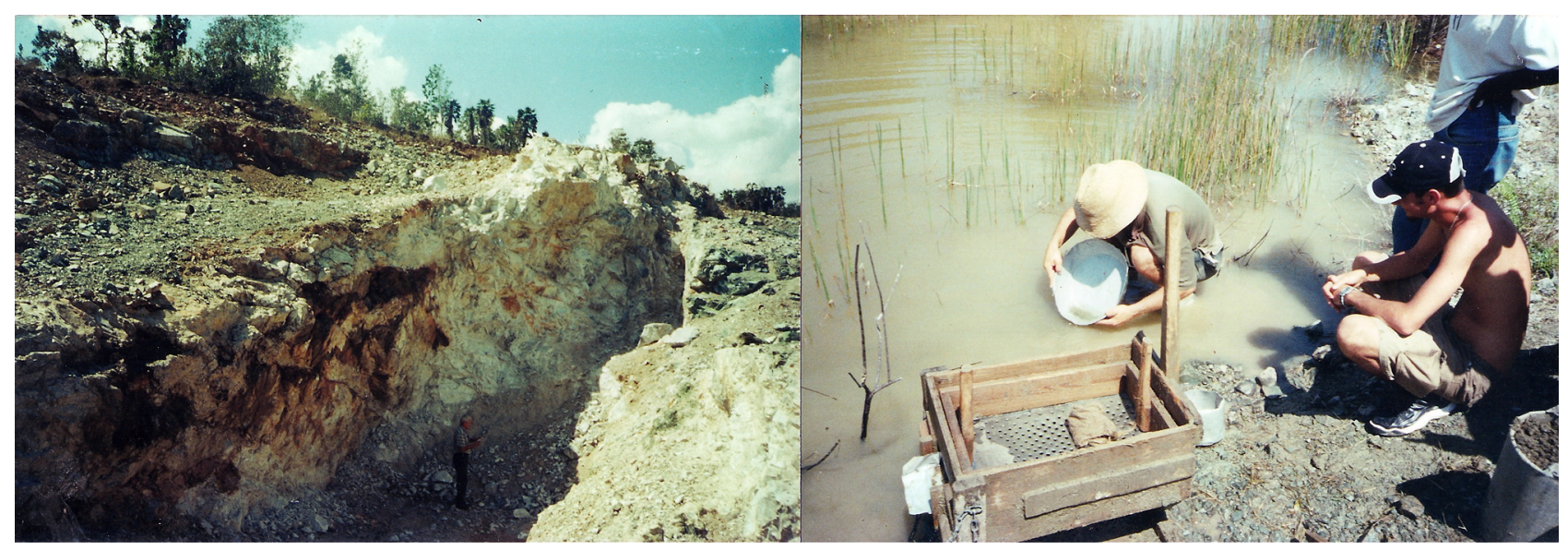

Figura 2. Bateador de la zona de Holguín. A la izquierda una trinchera de exploración del depósito Reina Victoria; a la derecha un jaguero concentrando oro a partir del lavado del escombro de las labores mineras de exploración.

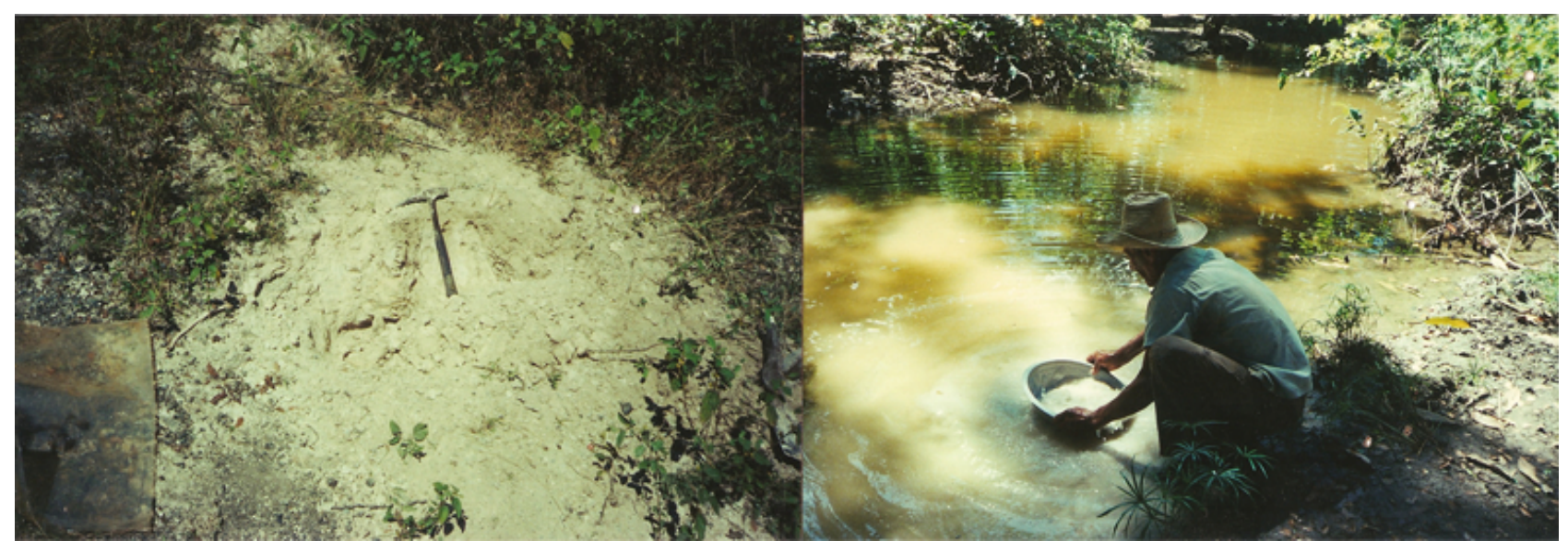

Figura 3. Bateador de la zona de Cuatro Palmas. Izquierda: Listvenita con oro. Derecha: Bateador en el Arroyo de Oro, Los Naranjos.

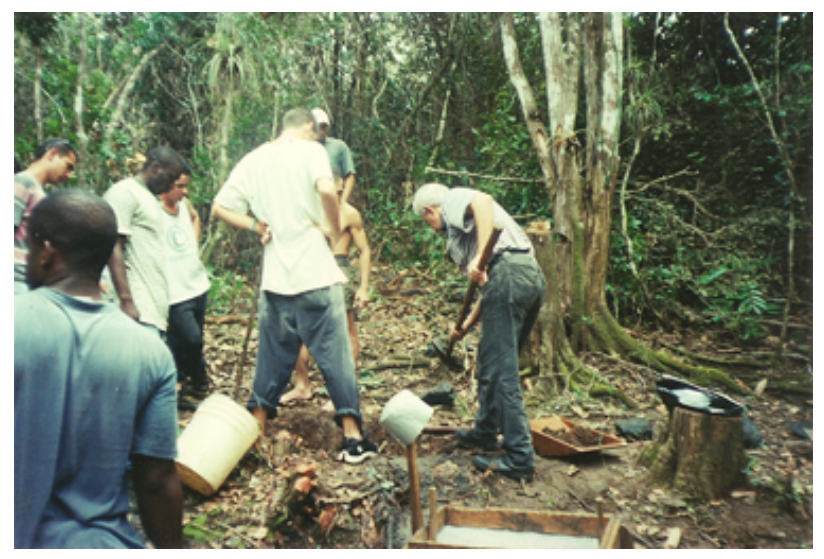

Figura 4. Minería artesanal de oro en los alrededores de la ciudad de Holguín. Estudiantes y profesores del Instituto Superior Minero Metalúrgico de Moa son testigos del impacto negativo que provoca esta actividad al medio ambiente.

a favor del potencial metalogenético del arco de islas volcánicas del Cretácico en Cuba.

\section{Mineralizaciones porfidicas de $\mathrm{Au}$-Cu}

En la región de Ciego de Ávila-Camagüey-Las Tunas han sido descritas mineralizaciones porfídicas de $\mathrm{Cu}-\mathrm{Au}$ con contenidos discretos de Mo, Ag y Au (Escobar, 1994; Lavandero y Bravo, 1994; Batista-González et al., 1998; Lugo-Primelles et al., 1998).

En el año 1996 se descubrió la mineralización porfídica de Au-Cu La Unión en Camagüey (Figura 5). La geoquímica de suelos desarrollados sobre porfiritas dioríticas arrojó valores anómalos de Au entre 30 y 3200 ppb. La zona mineralizada alcanza valores de hasta $3.53 \mathrm{~g} / \mathrm{t} \mathrm{Au}$. Los suelos que se desarrollan sobre el stockwork de cuarzo alcanza valores que oscilan entre 30 y $135 \mathrm{ppb}$. Las rocas huéspedes son dioritas cuarcíferas con contenidos de oro de hasta $1.18 \mathrm{~g} / \mathrm{t}$ (Alonso et al., 2009).

La mineralización se presenta de forma diseminada y en stockwork, compuesta principalmente por pirita y en menor grado por pirrotina, calcopirita, covelina, calcocina y esfalerita. Las alteraciones hidrotermales más comunes 


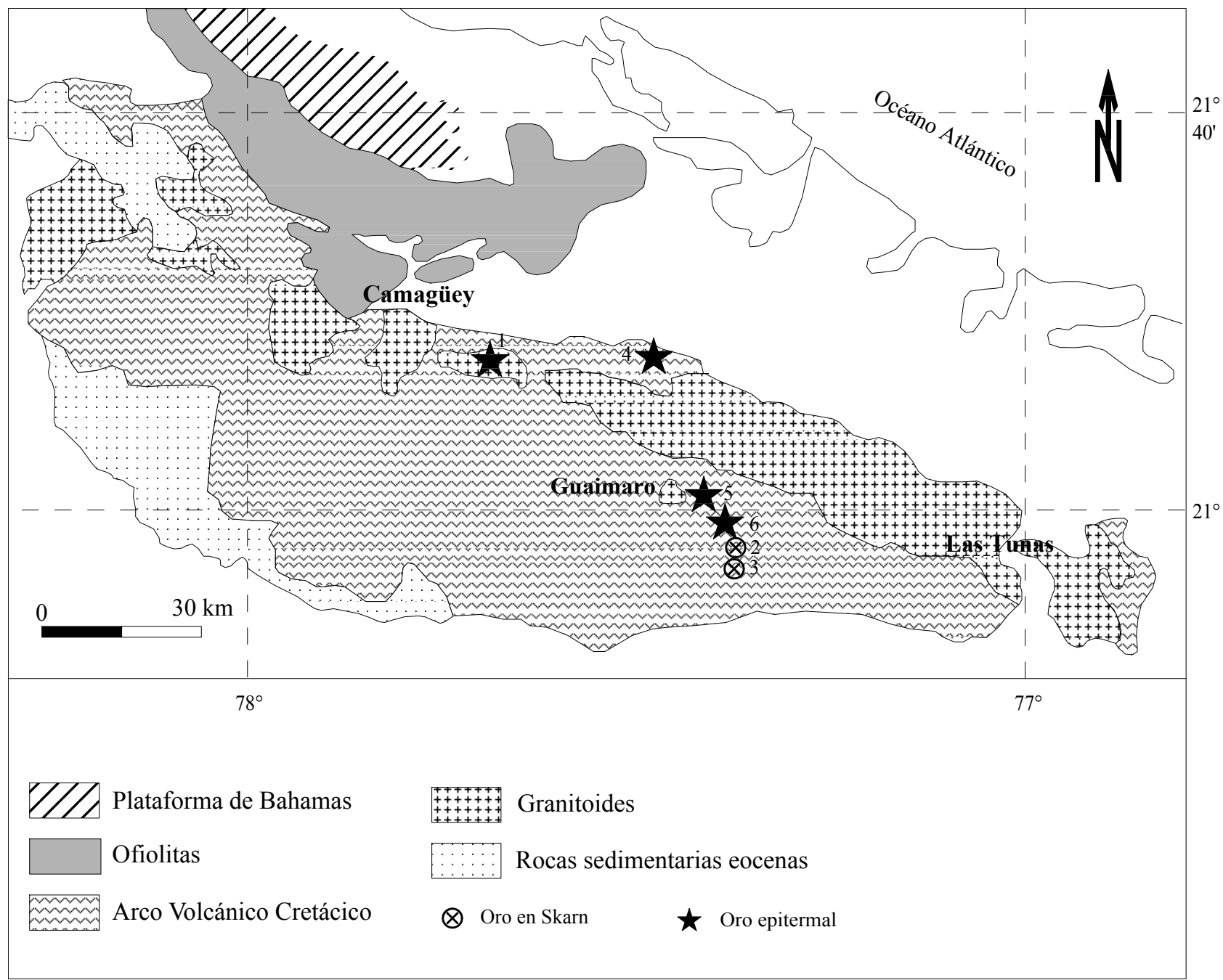

Figura 5. Depósitos de oro asociados al arco de islas volcánicas del Cretácico. Pórfidos de Au-Cu: 1- La Unión. Skarn de Au: 2- Georgina; 3- Abucha. Epitermales de oro de baja sulfuración: 4- Jacinto; 5- Florencia. Epitermal de alta sulfuración: 6- Golden Hill.

son la silicificación, carbonatización y sericitización. Las fases de alteración presentes son epidota, clorita, calcita y sericita (Alonso et al., 2009).

El oro se encuentra en forma nativa, formando granos globulares siempre asociado a la pirita, rellenando fracturas, diseminado o como oro invisible. El contenido de oro varía entre 1.09 y $3.53 \mathrm{~g} / \mathrm{t}$ (Alonso et al., 2009).

\section{Mineralizaciones de skarn de Au}

Estas mineralizaciones se desarrollan en la zona axial del arco de islas del Cretácico y son el resultado de procesos metasomáticos que han actuado sobre las rocas volcánicas y los propios intrusivos graníticos del arco. Las principales mineralizaciones de oro en skarn están distribuidas en la región de Camagüey-Las Tunas (Lavandero et al., 1988; Moreira et al., 2001).

Los skarn son epidóticos y se distribuyen dentro de una secuencia vulcanógena compuesta por tobas andesíticas y andesito-basálticas y lavas andesíticas y andesito-dacíticas, con intercalaciones de calizas de la Formación Crucero Contramaestre.

Los representantes típicos de estas mineralizaciones son los depósitos Georgina y Abucha (Figura 5). Dentro de su composición mineralógica se destacan la pirita, calcopirita, hematites, magnetita, bornita, melonita, calaverita, teluros, Au y Ag nativos. Los minerales de ganga son epidota, cuarzo y clorita. El contenido de oro varía entre 0.8 y $9.31 \mathrm{~g} / \mathrm{t}$, y la plata varía entre 1.6 y $36 \mathrm{~g} / \mathrm{t}$ (Moreira et al., 2001).

\section{Mineralizaciones epitermales de $\mathrm{Au}-\mathrm{Ag}$}

Las mineralizaciones epitermales de oro en Cuba se asocian exclusivamente al arco de islas volcánicas del Cretácico; su amplia distribución en la región de CamagüeyLas Tunas ubica esta porción del territorio nacional como la más prolífera en oro y a su vez la más perspectiva para proseguir estudios de exploración geológica (Kesler et al., 
2004). El sector más importante dentro de esta región es Guáimaro-Jobabo dado por la presencia de los depósitos epitermales de baja y alta sulfuración.

El depósito Jacinto es un típico epitermal de baja sulfuración. Se localiza a unos $60 \mathrm{~km}$ al este de la ciudad de Camagüey y a unos $25 \mathrm{~km}$ del poblado Guáimaro (Figura 5). Los trabajos de exploración ejecutados por la asociación económica internacional Carib Gold Exploration-Geominera S.A. lo descubren entre los años 1994-2001 (PimentelOlivera, 2009). Representa un sistema de 11 vetas, de las cuales las vetas Beatriz, El Limón y Sur Elena son las de mayor potencial aurífero (Simon et al., 1999).

La rocas huéspedes de las vetas de oro son brechas lávicas y lavas andesito-dacíticas, tobas de grano mediofino, propilitizadas y tectonizadas en diferente grado. Las vetas de oro están compuestas mayoritariamente por cuarzo, con menores cantidades de adularia, calcita hojosa, pirita y oro. La mineralización está compuesta por electrum y pirita, con cantidades subordinadas de calcopirita, esfalerita, galena y telururos de oro-plata. Según datos de CaribGold (Krason, 1999), los recursos estimados ascienden a 2.3 Mt de mena, con una ley de $5.83 \mathrm{~g} / \mathrm{t}$ de $\mathrm{Au}$.

El depósito Florencia (Figura 5) representa otro ejemplo de depósito epitermal de baja sulfuración asociado a este estadio metalogenético. Las rocas encajantes se componen de rocas porfídicas andesíticas y brechas lávicas, con menores intercalaciones de tobas y lentes de calizas. Las principales menas presentes son pirita, calcopirita, galena, esfalerita, magnetita, oro nativo y varios telururos de oro, plata, bismuto y plomo.

Los depósitos epitermales de alta sulfuración o tipo sulfato ácido se caracterizan por la presencia de caolinita y alunita como fases minerales presentes en la alteración argílica avanzada. Por otra parte, la presencia de sulfuros como covelina, calcosina, enargita y famatinita asociados con pirita indican un estado de sulfuración muy alto (Atkinson, 2003). En Cuba este tipo de mineralización reúne un gran número de mineralizaciones e indicios de oro con plata subordinada relacionados con la actividad volcánica de edad Aptiense-Campaniense. Ellos se localizan en el campo mineral Jobabo y su representante típico es Golden Hill (Barroso et al., 1998).

Este depósito se localiza a unos $8 \mathrm{~km}$ al N-NE del poblado de Jobabo, en la provincia de Las Tunas (Figura 5). Las principales menas presentes son pirita, enargita y oro con poca plata. Otros depósitos con características similares son: Loma Carolina, San Nicolás y El Pilar. Las alteraciones más frecuentes son la argílica avanzada, la argílica y la propilítica (Rodríguez-Romero, 2001).

\subsubsection{Estadio Campaniense tardio-Daniense temprano}

La mineralización aurífera de esta etapa está relacionada con ambientes de colisión (colisión tipo I, de antearcomargen continental pasivo) responsables de la amalgamación de las unidades continentales (Terreno Caribeana; GarcíaCasco et al., 2008) con el extinto arco de islas volcánicas del
Cretácico (Iturralde-Vinent, 1994; Iturralde-Vinent, 1997; Proenza y Melgarejo, 1998).

\section{Mineralizaciones de oro orogénico}

El ejemplo típico en Cuba es el yacimiento Delita (Lavandero et al., 1988; Bortnikov et al., 1989, 1993; López-Kramer et al., 1998; Proenza y Melgarejo, 1998). Este depósito se localiza al oeste de la Isla de la Juventud (Figura 6). Dichas mineralizaciones pudieron haberse originado durante la colisión de la margen continental con el arco de islas volcánico del Cretácico (vulcanitas de la Formación Sabana Grande). Según Groves et al. (1998) estos depósitos son considerados como tipo oro orogénico (véase Proenza y Melgarejo, 1998).

La mineralización se hospeda en rocas metamórficas de edad Jurásica (terreno Pinos) compuestas fundamentalmente por cuarcitas micáceas y esquistos cuarzo-micáceos (Bortnikov et al., 1989). El cuerpo mineral posee un espesor entre 0.2 y $0.5 \mathrm{~m}$ y está constituido por vetas de cuarzo con sulfuros dentro de rocas carbonosas, a los cuales se superpone una mineralización más tardía de carácter epitermal (Rodríguez-Vega y Díaz-Martínez, 2001). Las menas están constituidas por arsenopirita, pirita, sulfosales de antimonio, galena, esfalerita, calcopirita, vallerita y argentita, raramente marcasita, tetraedrita, pirrotina, electrum, wolframita, boulangerita, jamesonita, zinckenita, fülöppita, owyheeita, diaforita, andorita, pirargerita, plata y oro nativo. Los minerales no metálicos son cuarzo, caolinita, sericita, ankerita, zircón, zoisita, adularia y andalucita, y las texturas predominantes son vetítica, brechiforme, diseminada y paralela. La principal fase portadora de oro en el yacimiento Delita es la arsenopirita con contenidos de oro que varían entre 5.4 y 56 ppm (Bortnikov et al., 1993). El contenido promedio de oro en el yacimiento es de $5 \mathrm{~g} / \mathrm{t}$ (Rodríguez-Romero, 2001).

Las alteraciones acompañantes son sericitización, cuarcificación, carbonatización y piritización, mientras que los principales elementos y minerales acompañantes son $\mathrm{As}, \mathrm{Sb}, \mathrm{Ag}, \mathrm{Zn}, \mathrm{Cu},(\mathrm{W}),(\mathrm{Bi})$, sericita y minerales arcillosos. También se observa una zona de brecha formada por fragmentos de cuarzo y esquisto, siendo el conjunto cementado por cuarzo y sulfuros (Bortnikov et al., 1989).

En Cuba central han sido descritas numerosas manifestaciones de oro que pudieran estar relacionadas con procesos de colisión de los materiales de arco de islas del Cretácico y las ofiolitas septentrionales con el margen meridional de la plataforma de Bahamas (Figura 6).

\subsubsection{Estadio Daniense-Eoceno medio}

Durante este estadio en la parte occidental y central de Cuba se desarrollan cuencas sedimentarias transportadas, en las que se reconocen importantes unidades de olistostromas, que se relacionan con el emplazamiento tectónico de las ofiolitas. Al proceso de emplazamiento tectónico de las ofiolitas se vinculan importantes concentraciones de $\mathrm{Au}$ (Proenza y Melagrejo, 1998). En cambio, durante este 


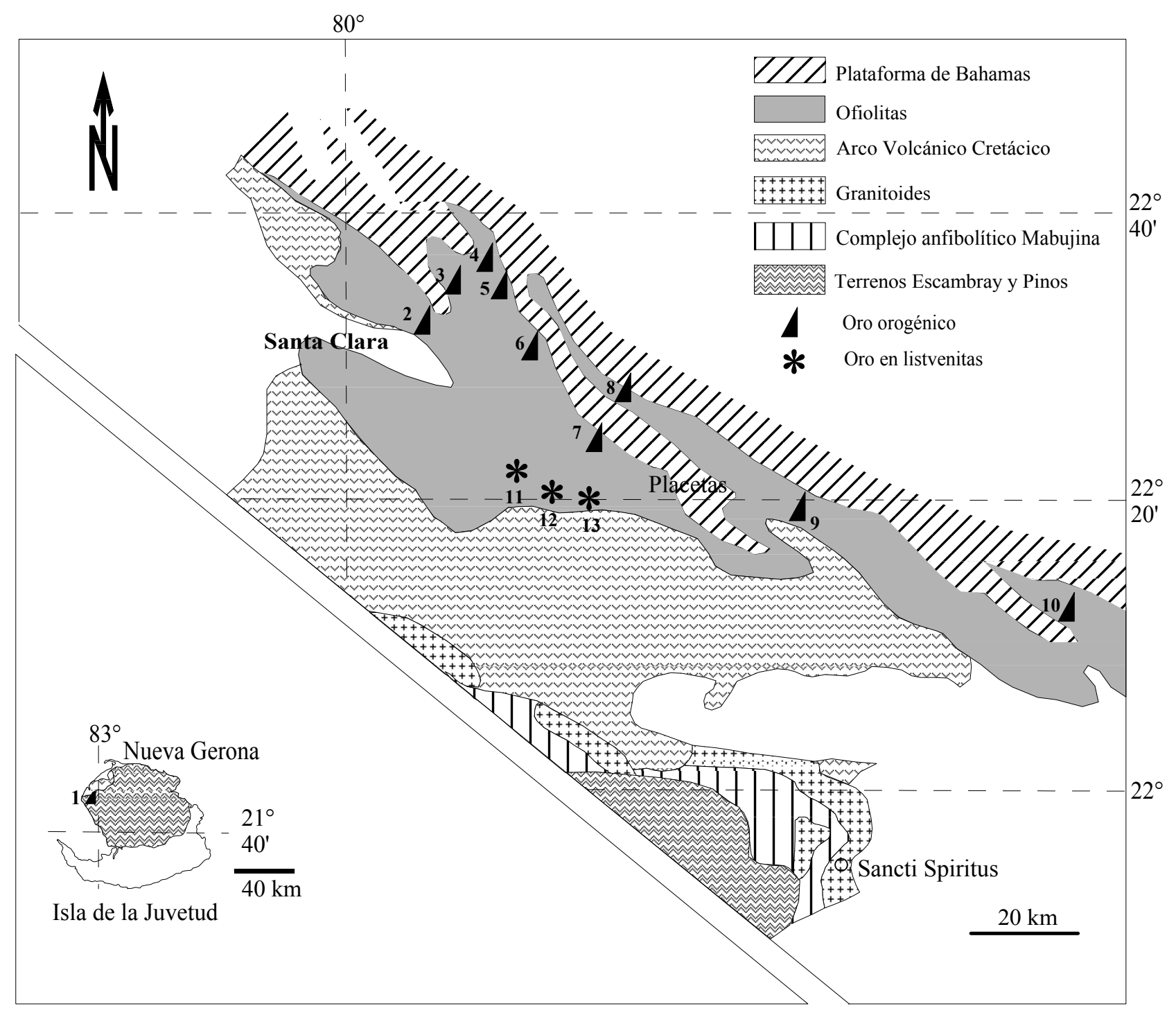

Figura 6. Mineralizaciones de oro asociadas a la colisión tipo I de edad Campaniense tardío-Daniense temprano (oro orogénico): 1- Delita; 2- San José; 3La Margarita; 4- San Pedro; 5- Carmita; 6- Los Cubanos; 7- Río Piedra; 8- Floridanos; 9- Pedro Barba; 10- Jobosí. Mineralizaciones de oro en Listvenitas del Daniense-Eoceno medio: 11- La Mascota; 12- Descanso; 13- Melonera

estadio en Cuba Oriental se registra una importante actividad volcánica asociada al denominado arco de islas volcánico Sierra Maestra, el cual contiene depósitos de Au.

\section{Mineralizaciones de oro en "listvenita"}

Estas mineralizaciones aparecen ampliamente distribuidas en Cuba central y en la región de Holguín. En general, la mineralización aurífera guarda relación espacial con las rocas propias de la asociación ofiolítica afectadas por procesos metasomáticos (listvenitización y rodingitización).

Las mineralizaciones auríferas de la región central de Cuba están representadas por los depósitos La Mascota, Descanso y Melonera (Figura 6) y representan las mineralizaciones auríferas en listvenitas más importantes y mejor conocidas de Cuba (Cabrera y Tolkunov, 1979;
Cabrera et al., 1986; Batista-González et al., 1998; LópezKramer et al., 1998; Proenza y Melgarejo, 1998).

Los cuerpos minerales se asocian espacialmente a zonas de fracturación y alteración metasomática de rocas ultrabásicas y básicas. La mineralización está representada por cuerpos metasomáticos de grano fino (listvenitas) y vetillas de cuarzo dentro de zona de esquistosidad y fallas poco definidas, en las cuales el oro se encuentra disperso en forma de granos muy finos. Dentro de las listvenitas también existen pequeñas cantidades de pirita, calcopirita, arsenopirita y esfalerita. El oro está presente en los sulfuros $\mathrm{y}$ en las listvenitas y como elementos acompañantes de esta mineralización se destacan As, Ag, Zn, Cu y Ni (Cabrera et al., 1986).

Los minerales metálicos lo constituyen sulfuros 
diseminados en forma de nidos y vetillas predominando la arsenopirita; en menor proporción aparecen galena, calcopirita, magnetita, ilmenita, cromita y sulfuros de hierro y níquel, y minoritariamente se encuentran la pirita, esfalerita, pirrotina y algunos telururos. El oro se presenta en forma nativa, electrum, altaita y cuproaurita. Sus contenidos varían desde los primeros gramos por tonelada hasta 1,850 $\mathrm{g} / \mathrm{t}$ y los de plata desde los primeros g/t hasta $100 \mathrm{~g} / \mathrm{t}$ (LópezKramer et al., 2006).

En la región de Holguín se conocen varios depósitos de oro en listvenitas, entre ellos los más importantes son Nuevo Potosí-Agrupada y Reina Victoria (Figura 7). Estos depósitos se localizan en extensas zonas de mineralizaciones diseminadas y vetítico-diseminadas en rocas ultrabásicas serpentinizadas, asociadas a cuerpos subvolcánicos y zonas de debilidades tectónicas generadas por fallas de sobrecorrimiento y transversales.

La piritización está difundida por todo el depósito,

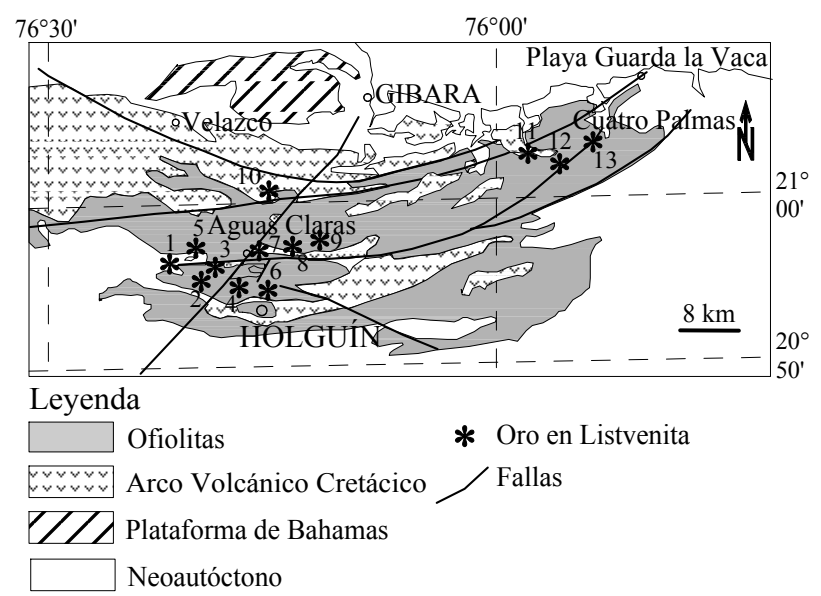

Figura 7. Depósitos de oro asociados al proceso de emplazamiento de las ofiolitas de edad Daniense-Eoceno medio. 1- Las Cuevas; 2- Reina Victoria; 3- Santiago; 4- Holguinera; 5- Tranquera; 6- La Ferrera; 7- Nuevo Potosí; 8- Agrupada; 9- La Fortuna; 10- Floro Pérez; 11- Campo Verde; 12- Cambute; 13- Cuatro Palmas. apareciendo en la mineralización diseminada y en la vetítica, afectando todas las litologías presentes.

Las zonas de mineralización diseminada forman extensas aureolas no asociadas a litologías específicas, con contenido de oro de 0.5 hasta $10 \mathrm{~g} / \mathrm{t}$. El oro aparece principalmente en forma nativa y en la arsenopirita, siendo de tamaño microscópico (López-Kramer et al., 2006).

\section{Mineralizaciones de oro epitermal (?)}

La metalogenia del arco de islas del Paleógeno es muy variada, lo que se hace evidente por la existencia de numerosas mineralizaciones de oro y plata. El ejemplo más importante y mejor conocido en Cuba es el depósito Oro Barita (Figura 8). Este depósito se ubica a $800 \mathrm{~m}$ al oeste del yacimiento El Cobre y ha sido caracterizado mediante una exploración detallada que culminó en el 2001 (Luna et al., 2003). Las alteraciones predominantes son la caolinitización y alunitización con procesos de argilitización avanzada.

El $28.20 \%$ de los granos de oro se encontraron asociados con la esfalerita, el $11.96 \%$ con la calcopirita, el $5.98 \%$ con pirita y el $23.93 \%$ en agregados complejos de dos o más sulfuros con o sin cuarzo. Por tanto, el $70.09 \%$ del total de granos de oro nativos hallados se encuentran en asociación con los sulfuros. En todos los casos los granos de oro observados se encontraron en las grietas y en los contactos entre los diferentes minerales (Luna, 2001; Luna et al., 2003).

La mineralización aurífera y auro-argentífera se relaciona con filones de cuarzo-sulfuros con presencia de vetillas de carbonatos. Esta mineralización puede enmarcarse dentro del estilo epitermal (Luna et al., 2003), aunque otros investigadores la han definido como vetas de cuarzo-sulfuro del tipo de baja sulfuración relacionadas con sistemas porfídicos (Rodríguez-Vega y Díaz-Martínez, 2001). En tal sentido es necesario continuar las investigaciones geoquímicas para definir un modelo genético más convincente sobre dichas mineralizaciones.

El depósito Oro Barita posee recursos evaluados ascendentes a 2.7 toneladas de oro con una ley promedio

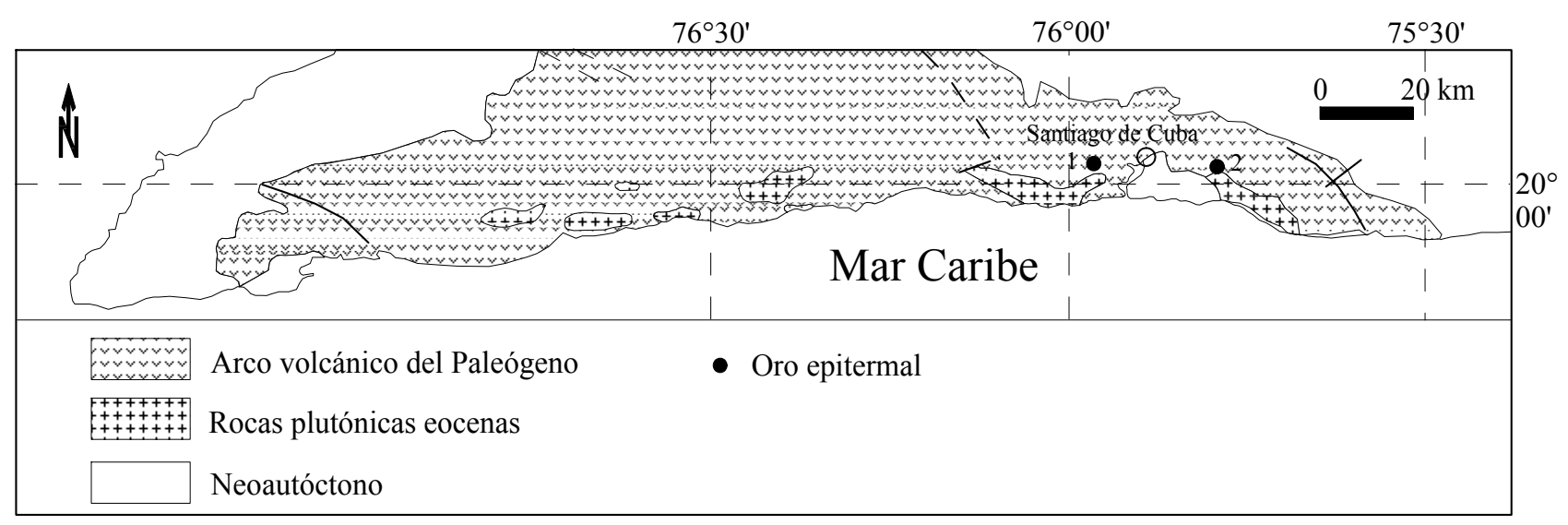

Figura 8. Mineralizaciones de oro asociadas al arco de islas volcánicas del Paleógeno. Epitermales: 1- Oro Barita; Manifestación Sigua. 
de $1.64 \mathrm{~g} / \mathrm{t}$ (Luna et al., 2003).

En los alrededores de este depósito se conocen las manifestaciones Melgarejo, Mina Nueva, Cobre Norte, Suroeste de Barita, Loma La Plata, Ermitaño, Ermitaño Este, La Esperanza y Santa Rosa. Los contenidos de oro oscilan entre 0.45 y $12.82 \mathrm{~g} / \mathrm{t}$. Al este de la ciudad de Santiago de Cuba se conoce la manifestación Sigua con contenidos de oro de hasta $90 \mathrm{~g} / \mathrm{t}$ (Luna et al., 2003).

\subsubsection{Estadio Oligoceno-Cuaternario ("Neoautóctono")}

Durante el estadio neo-platafórmico se dan condiciones favorables para la formación de importantes depósitos minerales de oro asociados a procesos de enriquecimiento supergénico en depósitos de sulfuros (sombreros de hierro) $\mathrm{y}$ procesos de concentración aluviales y marinos.

Mineralizaciones de oro en sombreros de hierro.

En Cuba, los sombreros de hierro originados a partir de la alteración supergénica de los depósitos de sulfuros tipo Sedex, VMS y epitermales, suelen ser ricos en Au y Ag. En la composición mineral de las menas de estos depósitos la pirita es el componente principal, lo cual garantiza la formación de potentes sombreros de hierro con oro libre. Tales mineralizaciones se han descrito en la parte occidental y oriental de Cuba.

En la provincia más occidental de la isla se localiza el yacimiento Oro Castellano, cuyo sombrero de hierro se exploró con una ley de corte de $1.5 \mathrm{~g} / \mathrm{t} \mathrm{Au}$.

\section{Mineralizaciones de placeres aluviales}

En Cuba, los placeres fluviales de oro con valor económico han sido descritos en la región Habana-Matanzas y en la región de Holguín. La productividad lineal de estos placeres varía entre 0.3 y $126 \mathrm{~kg}$ de oro $/ \mathrm{km}$ de río, con una media de $4.54 \mathrm{~kg}$ de oro por km de río (Morales-Quintana y Moreira, 1998).

En la región de Holguín se conocen aproximadamente 11 pequeños placeres aluviales, dentro de los cuales los de cauce y de terrazas son los más comunes (Almirante, Tranquera, Jobabo, Hicacos, Yabasito, Guajaney, Zapata, Guayacán, Bariay, Junucún y San Juan) con recursos evaluados en $539 \mathrm{~kg}$.

\section{Mineralizaciones de placeres marinos (laterales de playa)}

Los placeres laterales tipo playa han sido descritos en la región Sagua de Tánamo-Moa-Baracoa. Un ejemplo de depósito marino tipo playa con metales nobles lo constituye el placer Mejías (Díaz-Martínez, 1994; Díaz-Martínez et al., 1998; Proenza et al., 2004; Vila-Sánchez et al., 2004). Según estos autores el placer tiene forma de capas alargadas, con yacencia casi horizontal y un rumbo paralelo a la costa. La mineralización se compone de magnetita, titanomagnetita, cromita, óxidos de titanio, partículas de oro nativo, de electrum y de oro mercurial, así como de minerales del grupo del platino (laurita, irarsita, erlichmanita).

\subsection{Perspectiva de la minería del oro en Cuba}

$\mathrm{Al}$ inventariar las mineralizaciones e indicios de oro en Cuba (más de 100) queda demostrado el potencial aurífero de la isla. Sin embargo, los problemas ambientales que producen las explotaciones a cielo abierto, la incidencia de la metalurgia sobre el ambiente, los bajos contenidos de oro en las menas y los reducidos tonelajes de mineral industrial, tanto para las mineralizaciones primarias como secundarias, limitan significativamente cualquier intento de despegue de esta importante actividad económica.

Actualmente algunos depósitos de oro conocidos cuentan con estudios de factibilidad y en las nuevas condiciones económicas del país y los precios del metal en el mercado internacional pueden ser explotados de manera racional. Por ejemplo, el yacimiento Oro Barita, con recursos estimados de 2.7 toneladas y un contenido promedio de $1.64 \mathrm{~g} / \mathrm{t}$ de oro. Se prevé además la explotación del sombrero de hierro del yacimiento Golden Hill perteneciente al campo mineral Jobabo.

Algunos criterios que sustentan un promisorio futuro para la minería del oro en Cuba son:

1. La inmensa mayoría de los depósitos de cobre y metales base de Cuba son portadores de oro.

2. Numerosos depósitos de oro y plata de tipo skarn, pórfidos y epitermales asociados al arco de islas volcánicas del Cretácico poseen grandes perspectivas para ser evaluados y explotados en un futuro no muy lejano.

3. En la región central y oriental de Cuba se conocen mineralizaciones de oro de tipo listvenitas en ofiolitas que han sido explotados desde el tiempo de la colonia.

4. Existen en la isla numerosos pequeños placeres aluviales y laterales de playa que pueden contribuir significativamente al balance aurífero de la nación.

5. Los sombreros de hierro de muchos yacimientos piríticos con metales base en la parte occidental y central de la isla están enriquecidos en oro libre cuya extracción puede hacerse en condiciones ventajosas.

\section{Conclusiones}

En la evolución geológica de Cuba se reconocen 4 episodios metalogenéticos principales de oro. i) El AptienseCampaniense, con la formación de mineralizaciones porfídicas de $\mathrm{Au}-\mathrm{Cu}$, de skarn de $\mathrm{Au}$ y epitermales de Au y Ag de baja y alta sulfuración; ii) el estadio Campaniense tardío-Daniense temprano, con el desarrollo de mineralizaciones de oro de tipo orogénico; iii) un estadio Daniense-Eoceno medio, muy prolífero en mineralizaciones de oro en listvenitas en Cuba central y en el arco de islas volcánicas del Paleógeno; y iv) Oligoceno-reciente, donde se han originado las mineralizaciones de oro en sombreros de hierro y los depósitos de placeres. La futura minería del oro en Cuba es muy atractiva, pero a pequeña y mediana 
escala, dada las dimensiones de sus recursos auríferos..

\section{Agradecimientos}

Este trabajo es una contribución a un proyecto internacional de cooperación al desarrollo de la Universidad de Barcelona (Vicerectorat de Política i Mobilitat). Este trabajo ha sido mejorado gracias a los comentarios y sugerencias de Joaquín Proenza y Joan Carles Melgarejo.

\section{Referencias}

Alonso, J., Ulloa, M., Donet, P., 2009, La unión, una manifestación muy perspectiva de $\mathrm{Au} \pm \mathrm{Cu}$ de tipo pórfido (resumen), en III Convención de Ciencias de la Tierra: La Habana, Cuba, Sociedad Cubana de Geología, 1-8.

Ariosa-Iznaga, D.J., Díaz-Martínez, R., 2001, Modelos de yacimientos minerales: tipologías y aplicaciones: Revista Minería y Geología, 18, 3-14.

Ariosa-Iznaga, D.J., 2002, La modelación descriptiva de yacimientos minerales en Cuba: Moa, Cuba, Instituto Superior Minero Metalúrgico de Moa, Tesis doctoral, $103 \mathrm{p}$.

Atkinson, W., 2003, Geoquímica de los depósitos de oro: evidencias geológicas, modos de transporte hidrotermal para la depositación de oro y sus aplicaciones en la exploración: Cajamarca, Perú, Universidad de Cajamarca, Taller Internacional.

Barroso, A., Lugo-Primelles, R., Torres-Domínguez. A., 1998, Nuevos resultados geólogo-geofísicos amplían las perspectividades de mineralización del yacimiento Golden Hill (Resumen), en III Congreso Cubano de Geología y Minería: La Habana, Cuba, Sociedad Cubana de Geología, 49-50.

Batista-González, R., Montano, J.L., Martínez, J. Pérez-Nevot, N., 1998, Mapa de los recursos minerales. Cuba Central a escala 1: 100000 (Resumen), en III Congreso Cubano de Geología y Minería, Cuba, Sociedad Cubana de Geología, 53-55.

Bortnikov, N.S., Kramer, K. L., Genkin, A.D., Kul'nev, A.S., 1989, The mineralogy and conditions of formation of the Delita gold deposit, Cuba: International Geology Review, 31, 1158-1172.

Bortnikov, N.S., Genkin, A.D., Chryssoulis, S., 1993, Deposition environment of gold-bearing arsenopiryte in mesothermal deposits, in Fenoll Hach-Ali, F., Torres-Ruiz, J., Gervilla, F. (eds.), Current Research in Geology Applied to ore Deposits: Granada, España, SGA, 45-48.

Brauns, R., 1860, Mineralogía, Biblioteca General, Universidad de La Habana, 549-B.

Cabrera, R., Kramer, J.L., Dobrovolskaya, M., Catá, A., 1986, La formación menífera auro-listvenítica del yacimiento Descanso en Villa Clara (Cuba): Revista Ciencias de la Tierra y del Espacio, 11, 59-66.

Cabrera, R., Tolkunov, A.E., 1979, Tipos y condiciones geológicas de formación de los yacimientos de oro de la zona mineral septentrional de la antigua provincia de Las Villas: Revista Ciencias de la Tierra y del Espacio, 1, 51-68.

Calvache-Dorado, A., 1944, Historia y desarrollo de la minería en Cuba: La Habana, Neptuno, 170 p.

Capote-Marrero, G., Cruz-Pacheco, M.S., González, D., Altarriba, I., Bravo, F., Carrillo, D., De La Nuez, D., Cazañas, X., 2009, El arco Cretácico del territorio Ciego-Camagüey-Las Tunas, a escala 1:100.000 (resumen), en III Convención de Ciencias de la Tierra: La Habana, Sociedad Cubana de Geología, 1-22.

Díaz-Martínez, R., 1994, Sobre la existencia de placeres laterales en el nordeste de Cuba Oriental (resumen), en Segundo Congreso Cubano de Geología y Minería: Santiago de Cuba, Sociedad Cubana de Geología, 112.
Díaz-Martínez, R., Proenza, J.A., Comas, J., Fernández-Bellón, O., Fabra, J.M., Guinart, O., Melgarejo, J.C., 1998, El placer lateral de playa Mejías (noreste de Cuba Oriental): un ejemplo de interacción de procesos aluviales y marinos en la concentración de minerales de elementos preciosos: Acta Geologica Hispanica, 33, 351-372.

Díaz de Villalvilla, L., Dilla, M., 1985, Proposición para una división de la llamada formación tobas (Provincia Cienfuegos, Villa Clara y Sancti Spiritus): Revista Serie Geológica, 1, 133-154.

Donnelly, T.W., Beets, D., Carr, M.J., Jackson, T., Klaver, G., Lewis, J.F., Maury, R., Schellenkens, H., Smith, A.L., Wadge, G., Westercamp, D., 1990, History and tectonic setting of Caribbean magmatism, in Dengo, G., Case, J.E. (eds.), The Caribbean region: Boulder, Colorado, Geolocical Society of America, The Geology of North America, H, 339-374.

Escobar, E., 1994, Mineralizaciones de metales básicos y preciosos, asociados al arco volcánico Cretácico en la región Ciego de Ávila-Camagüey-Tunas (resumen), en Segundo Congreso Cubano de Geología y Minería: Santiago de Cuba, Sociedad Cubana de Geología, 96.

Font-Cruz, L.R., 1985, Proyecto de exploración de explotación del yacimiento Nuevo Potosí, Aguas Claras: Moa, Cuba, Instituto Superior Minero Metalúrgico, Trabajo de diploma, $60 \mathrm{p}$.

García-Casco, A., Iturralde-Vinent, M.A., Pindell, J., 2008, Latest Cretaceous collision/accretion between the Caribbean Plate and Caribeana: origin of metamorphic terranes in the Greater Antilles: International Geology Review, 50, 781-809.

Groves, D.I., Goldfarb, R.J., Gebre-Mariam, M., Hagemann, S.G., Robert, F., 1998, Orogenic gold deposits: A proposed classification in the context of their crustal distribution and relationship to other gold deposit types: Ore Geology Reviews, 13, 7-27.

Hamilton, L., 1998, Grado de estudio geológico de los yacimientos de oro en Cuba: Moa, Cuba, Instituto Superior Minero Metalúrgico, Trabajo de diploma, $66 \mathrm{p}$.

Iturralde-Vinent, M.A., 1994, Cuban Geology: A new plate tectonic synthesis: Journal of Petroleum Geology, 17, 39-69.

Iturralde-Vinent, M.A., 1997, Introducción a la geología de Cuba, en Furrazola, G.F., Núñez, K.E., (eds.), Estudios sobre la geología de Cuba: La Habana, Cuba, Centro Nacional de Información Geológica, 35-68.

Iturralde-Vinent, M.A., 1998, Sinopsis de la constitución geológica de Cuba: Acta Geológica Hispánica, 33, 9-56.

Kesler, S.E., Hall, C.M., Russell, N., Piñero, E., Sánchez, C.R., Pérez, M.R., Moreira, J., 2004, Age of the Camagüey gold-silver district, Cuba: tectonic evolution and preservation of epithermal mineralization in volcanic arcs: Economic Geology, 99, 869-886.

Krason, J., 1999, Cuba: Mining Journal, 3, 126-127.

Lavandero, R.M., Bravo, F., 1994, Ambientes geodinámicos y su relación con los yacimientos minerales metálicos en la isla de Cuba (resumen), en Segundo Congreso Cubano de Geología y Minería: Santiago de Cuba, Sociedad Cubana de Geología, 97.

Lavandero, R.M., Estrugo, M., Santa Cruz-Pacheco, M., Bravo, F., Melnikova, A.A., Cabrera, R., Trofinov, V.A., Romero, J., Altarriba, I., Álvarez, P., Aniatov, I.I., Badamgavin, B., Barishev, A.N., Carrillo, D.J., Cazañas, X., Cuellar, N., Dovbnia, A.V., Formell, F., García, M., González, D., Gue, G.G., Janchivin, A., Krapiva, I.J., López, J., Lozanov, I., Montenegro, J., Pantaleón, G., Stefanov, N., Vázquez, O., Zaagoskin, A.M., Zhidkov, A.Y., 1988, Mapa de yacimientos minerales metálicos y aguas minerales de la República de Cuba, Escala 1:500 000, Ciudad de la Habana, Cuba, 4 hojas.

Lavandero, R.M., Moreira, J., Torres, J.L., Suárez, A., Montano, J., Morales, A., Altarriba, I., Bravo, F., Echevarría, B., Carrillo, D., Chang, J.L., González, D., Pantaleón, G., 2001, Potencialidades de recursos minerales para metales preciosos y base en la región oriental de Cuba (resumen), en IV Congreso Cubano de Geología y Minería: La Habana, Cuba, Sociedad Cubana de Geología, 1-10.

Lebron, M.C., Perfit, M.R., 1993, Stratigraphic and petrochemical data support subduction polarity reversal of the Cretaceous caribbean island arc: Journal of Geology, 101, 389-396. 
López-Kramer, J., Moreira, J., Pantaleón, G.J., Lavandero, R.M., Montano, J., Cruz-Martín, J., 1998, Tipos mineralógicos de algunos yacimientos auríferos de Cuba (resumen), en III Congreso Cubano de Geología y Minería: La Habana, Cuba, Sociedad Cubana de Geología, 371-374.

López-Kramer, J., Moreira-Martínez, J.M., Vera-Blanco, J., PachecoCabrera, A., Toujagne de la Rosa, R., 2006, Nota técnica mineralogía, geoquímica y algunas medidas de protección ambiental en yacimientos de oro de Cuba: Revista Universidad, Ciencia y Tecnología, 10, 301-106.

Lugo-Primelles, R., Barroso, A., Lugo, R., Escobar, E., 1998, Geología y metalogenia del campo mineral Guáimaro. Enfoque actual (resumen), en III Congreso Cubano de Geología y Minería: La Habana, Cuba, Sociedad Cubana de Geología, 390-393.

Luna, A., 2001, Perspectivas de mineralización aurífera en la región El Cobre (resumen), en IV Congreso cubano de geología: La Habana, Cuba, Sociedad Cubana de Geología, 172-176.

Luna, A., Medina-Batista, A., Falero-Salgado, R., 2003, Resultados de los trabajos de exploración en el depósito Oro Barita y las perspectivas de encontrar nuevos recursos (resumen), en V Congreso Cubano de Geología: La Habana, Cuba, Sociedad Cubana de Geología, 32-40.

Morales-Quintana, A.R., Moreira, J., 1998, Mapa metalogenético pronóstico de los placeres de oro en la región de Habana-Matanzas (resumen), en III Congreso cubano de geología y minería: La Habana, Cuba, Sociedad Cubana de Geología, 470-473.

Moreira, J., Torres, J.L., Montano, J., Lavandero, R., Sánchez, R., Cazañas, X., 2001, Depósitos de skarn de Cuba (resumen), en III Congreso Cubano de Geología y Minería: La Habana, Cuba, Sociedad Cubana de Geología, 91-109.

Pimentel-Olivera, H., Piñero-Pérez, E., Balvis-Filiberto, C., 2009, Actualización de la evaluación geólogo-económica para la veta aurífera Beatriz (resumen), en III Convención de Ciencias de la Tierra: La Habana, Cuba, Sociedad Cubana de Geología, 21.

Proenza, J.A., Melgarejo, J.C., 1998, Una introducción a la metalogenia de Cuba bajo la perspectiva de la tectónica de placas: Geologica Acta, 33, 89-131.
Proenza, J.A., Rodríguez-Vega, A., Díaz-Martínez, R., Gervilla, F., Melgarejo, J.C., Ramayo, L., Vila, A.R., 2004, Distribución de elementos del grupo del platino (EGP) y Au en la faja ofiolítica Mayarí-Baracoa (Cuba oriental), en Pereira, E.S., Castroviejo, R., Ortiz, F. (eds.), Complejos ofiolíticos en Iberoamérica: guías de prospección para metales preciosos: Madrid, Proyecto XIII.1Ciencia y Tecnología Para el Desarrollo, 309-336.

Rodríguez-Romero, M., 2001, Clasificación tipológica de los depósitos de oro en Cuba (resumen), en III Congreso Cubano de Geología y Minería: La Habana, Cuba, Sociedad Cubana de Geología, 188-197.

Rodríguez-Vega, A., Díaz-Martínez, R., 2001, La mineralización aurífera en Cuba, en Espí, J.A. (ed.), El libro de la minería del Oro en Iberoamérica: Madrid, CYTED, 249-264.

Sánchez-Rodríguez, S.M., 2001, La socioeconomía de la minería del oro, en Espí, J.A. (ed.), El libro de Minería del Oro en Iberoamérica: Madrid, CYTED, 53-81.

Simon, G., Kesler, S.E., Russell, N., Hall, C.M., Bell, D., Pinero, E., 1999, Epithermal gold mineralization in an old volcanic arc: the Jacinto deposit, Camaguey District, Cuba: Economic Geology, 94, 487-506.

Soto-González, L.D., 1981, Apuntes sobre la historia de la minería cubana: Santiago de Cuba, Editorial Oriente, 121p.

Torres-Cuevas, E., Loyola-Vega, O., 2001, Historia de Cuba 1492-1898. Formación y liberación de la nación: La Habana, Cuba, Editorial Pueblo y Educación, $404 \mathrm{p}$.

Vila-Sánchez, A.R., Díaz-Martínez, R., Proenza, J.A., Melgarejo, J.C., 2004, Caracterización morfológica, textural y composicional de las partículas de oro reveladas en placeres marinos de las playas Mejías y Jiguaní (NE de Cuba Oriental): Geociencias, 23, 43-53

Manuscrito recibido: Diciembre 2, 2009

Manuscrito corregido recibido: Enero 7, 2010.

Manuscrito aceptado: Enero 30, 2010. 